\title{
Treatment Strategies for Metastatic Castration-Sensitive Prostate Cancer: From "All-Comers" to "Personalized" Approach
}

\author{
Kenichi Harada' \\ Masaki Shiota ${ }^{2}$ \\ Akinori Minato ${ }^{3}$ \\ Masahiro Matsumoto ${ }^{3}$ \\ Ikko Tomisaki ${ }^{3}$ \\ Masato Fujisawa' \\ Naohiro Fujimoto (D) $^{3}$ \\ 'Department of Urology, Kobe \\ University Graduate School of \\ Medicine, Kobe, 650-0017, Japan; \\ ${ }^{2}$ Department of Urology, Graduate \\ School of Medical Sciences, Kyushu \\ University, Fukuoka, 8I2-8582, Japan; \\ ${ }^{3}$ Department of Urology, University of \\ Occupational and Environmental \\ Health, Kitakyushu, 807-8556, Japan
}

Correspondence: Naohiro Fujimoto Department of Urology, University of Occupational and Environmental Health, I-I Iseigaoka, Yahatanishi-Ku, Kitakyushu, 807-8556, Japan

Tel +81936917446

Fax +81936038724

Email n-fuji@med.uoeh-u.ac.jp

\begin{abstract}
Standard treatment for metastatic castration-sensitive prostate cancer (mCSPC) was androgen-deprivation therapy (ADT) for $>7$ decades, and this was termed the "allcomers" approach. A remarkable evolution in the treatment of $\mathrm{mCSPC}$ has been noted in the previous several years. High-quality clinical trials have shown that the addition of docetaxel or androgen receptor pathway inhibitors, such as abiraterone acetate, enzalutamide, and apalutamide, to ADT improves the overall survival (OS) as compared to ADT alone. The first 2 trials demonstrated the benefits of docetaxel and abiraterone acetate in terms of OS in high-volume and high-risk cancer subgroups, respectively. The later trials indicated that upfront combination therapies were associated with improved OS in all patients, irrespective of tumor volume and risk category. Upfront combination therapies are becoming a standard of care for all patients with mCSPC. However, meta-analyses have failed to show that all upfront combination therapies provide significant survival benefits in all patient subgroups. In the low-volume subgroup, significance was observed only for treatment with enzalutamide and radiation to the prostate. Men with low-volume low-risk cancer who have favorable response to ADT achieve long-term survival with ADT only, and toxicities induced by combination therapies would exceed the benefit for these patients. Treatments should be tailored to each patient because mCSPC has marked diversity in its biological and clinical features. Recent advances in diagnostic and molecular technologies will provide useful prognostic and predictive biomarkers, and the treatment strategy will shift from the "for all-comers" to the "individualized" approach.
\end{abstract}

Keywords: castration-sensitive, prostate cancer, personalized therapy, biomarker, treatment decision

\section{Introduction}

Prostate cancer is one of the most common cancers in the male population. As of 2020 , the estimated number of new cases of prostate cancer is 191,930 , and this cancer represents the most commonly diagnosed cancer in the United States. ${ }^{1}$ The number of patients with prostate cancer has been increasing worldwide, particularly in Asia and developing countries. ${ }^{2}$ The global incidence of prostate cancer is expected to rise further with an increase in the elderly population. Prognosis of localized prostate cancer is favorable, and the 5-year relative survival rate is $>99 \%$; however, the 5-year relative survival rate for metastatic prostate cancer is only $30 \%{ }^{1}$ For metastatic prostate cancer, androgen-deprivation therapy (ADT) with either surgical or medical castration was the only standard of care. ADT was 
recommended as the primary treatment for all patients with metastatic castration-sensitive prostate cancer (mCSPC), irrespective of patient and cancer characteristics, and it was a treatment for "all-comers". Up to $95 \%$ of patients respond to ADT; however, almost all patients progress to fatal disease, castration-resistant prostate cancer (CRPC). ${ }^{3}$ A recent clinical trial, Systemic Therapy in Advancing or Metastatic Prostate Cancer: Evaluation of Drug Efficacy (STAMPEDE), revealed that the median failure-free survival (FFS) and OS for men with mCSPC receiving $\mathrm{ADT}$ were 11 mon and 42 mon, respectively, even though this trial recruited relatively younger patients without serious complications than those in the actual clinical setting. ${ }^{4}$

The advent of novel therapeutic strategies is changing the treatment paradigms. Large-scale clinical trials have demonstrated that upfront combination therapies using chemotherapeutic agent docetaxel and androgen receptor pathway inhibitors (ARPIs) achieve better survival outcomes than ADT alone. $^{5-11}$ The Chemohormonal Therapy Versus Androgen Ablation Randomized Trial for Extensive Disease in Prostate Cancer (CHAATED) trial and the Abiraterone Acetate Plus Prednisone in Patients With Newly Diagnosed High-Risk Metastatic CastrationSensitive Prostate Cancer (LATITUDE) trial showed the survival benefit of upfront combination therapies in the subgroup defined by tumor burden and cancer risk, respectively. ${ }^{5,7}$ However, more recent studies have shown that combination therapies improved survival in all patients, irrespective of tumor burden and risk. ${ }^{9-12}$ Thus, upfront combination therapies are becoming the first-line standard of care for "all-comers" as per the recommendations of recent clinical guidelines. ${ }^{13,14}$ This direction may go against the recent general trend of cancer treatment because cancer treatment is advancing from the "allcomers" approach to the "individualized" and "precision" medicine approach.

Previous review papers have described the details of the clinical trials for mCSPC. ${ }^{15-20}$ Thus, in the present review, we discuss the primary treatment for $\mathrm{mCSPC}$ from the viewpoint of personalized treatment choice.

\section{From "All-Comers" to "Personalized" Treatment}

As mentioned above, ADT with or without 1st generation antiandrogens such as bicalutamide and flutamide were the only standard treatment for all $\mathrm{mCSPC}$ patients before the important results of the clinical trial, CHAATED, was published. $^{5}$ This large-scale Phase 3 study demonstrated that adding six cycles of docetaxel to ADT as a primary treatment achieved better OS than the administration of ADT alone. A long-term follow-up study with a median follow-up duration of 53.7 mon confirmed the results. ${ }^{21}$ The OS benefit of upfront docetaxel over ADT alone was observed in patients with a high-volume metastatic burden [median OS: 51.2 vs 33.4 mon; hazard ratio (HR), 0.72; $95 \%$ confidence interval $(\mathrm{CI}), 0.59-0.89 ; \mathrm{p}=0.0018]$, but not in those with low-volume disease (median OS: 63.5 mon vs not reached; HR, 1.04; 95\% CI, 0.70-1.55; p = 0.86). In this trial, high-volume was defined as visceral metastases and/or $\geq>4$ bone metastases and at least one outside the vertebral column and pelvis. ${ }^{5}$ Analysis of the aggregate data of 2 independent clinical trials, CHAATED and Androgen-deprivation therapy alone or with docetaxel in non-castrate metastatic prostate cancer (GETUGAFU15), also showed that upfront docetaxel was able to achieve better OS than ADT alone in patients with highvolume (HR, 0.68; 95\% CI, 0.56-0.82; p < 0.001), but not in those with low-volume disease (HR, 1.03; 95\% CI, $0.77-1.38 ; \mathrm{p}=0.8)^{22}$

Another clinical trial LATITUDE demonstrated the benefit for OS by using a combination of abiraterone acetate, selective CYP17 inhibitor, plus prednisone (AAP), and ADT in patients with high-risk mCSPC (median OS; not reached vs 34.7 mon, HR, 0.66; 95\% CI, $0.57-0.78 ; \mathrm{p}<0.0001) .^{7,23}$ In this trial, high-risk was defined as the presence of at least two of the following three factors; Gleason score $\geq 8, \geq 3$ bone metastases, and visceral metastases. These results indicate that patients with a high-volume metastatic burden and high-risk cancer should be treated with upfront docetaxel and AAP, respectively, and the standard treatment has changed from the "all-comers" to the "personalized" approach.

\section{From "Personalized" to "All- Comers"}

A systematic review and meta-analyses of the aggregate data of the CHAARTED, GETUG-AFU15, and STAMPEDE trials indicated that the upfront use of docetaxel showed better OS in patients with $\mathrm{mCSPC}$ than in those with ADT alone (HR, 0.77; 95\% CI, 0.68-0.87; $<<0.0001) .{ }^{24}$ As per the STAMPEDE trial, upfront docetaxel improved the OS irrespective of the metastatic tumor burden (HR, 0.81; 95\% CI, 0.69-0.95; $\mathrm{p}=0.003) .{ }^{25}$ For low-volume patients, the median OS was 
93.2 mon and 76.7 mon for upfront docetaxel and ADT alone, respectively (HR, 0.76; 95\% CI, 0.54-1.07). This HR was consistent with that in high-volume patients (HR, 0.81, 95\% CI, 0.64-1.02), suggesting that upfront docetaxel would be beneficial for all patients with $\mathrm{mCSPC}$, irrespective of the metastatic burden, and this treatment may be for "all-comers".

In addition, the efficacy of upfront AAP was assessed in the STAMPEDE trial that recruited $\mathrm{mCSPC}$ patients irrespective of tumor volume and risk. Patients in the trial arm $G$ and the arm A received AAP + ADT and ADT alone, respectively. ${ }^{12}$ The OS benefit of upfront $\mathrm{AAP}+\mathrm{ADT}$ over ADT alone was achieved not only in high-risk patients (absolute 3-year survival: 65\% vs 45\%; HR, 0.54; 95\% CI, 0.41-0.70), but also in low-risk patients (absolute 3-year survival: $83 \%$ vs 78\%; HR, $0.66,95 \%$ CI, 0.44-0.98). AAP +ADT was also associated with more favorable FFS, which was defined as radiological, clinical, or PSA progression, or death from prostate cancer, than ADT alone in low-risk (HR, 0.25; 95\% CI, $0.17-0.33$ ) and high-risk patients (HR, 0.31; 95\% CI, $0.25-0.39$ ). The heterogeneity of OS and FFS between high- and low-risk groups was not significant. As per the CHAATED volume definition, ${ }^{5}$ AAP + ADT improved the OS and FFS more than ADT alone in both, high-volume (HR for OS, 0.60, 95\% CI, 0.46-0.78, HR for FFS, 0.33 , 95\% CI, 0.26-0.41) and low-volume (HR for OS, 0.64; 95\% CI, 0.42-0.97, HR for FFS, 0.26; 95\% CI, 0.19-0.36) subgroups. In addition, AAP + ADT conferred a more significant improvement in the OS and FFS than ADT alone in the low-risk low-volume (double low) subgroup (HR for OS, 0.56; 95\% CI, 0.34-0.94) and FFS (HR, 0.21; 95\% CI, 0.14-0.30). These results suggest that upfront AAP would improve the treatment outcomes in patients with $\mathrm{mCSPC}$, irrespective of the tumor burden or risk and may be the standard of care for "all-comers".

Enzalutamide plus Androgen-Deprivation Therapy for Metastatic Hormone-Sensitive Prostate Cancer (ARCHES), a randomized Phase III study, compared the clinical benefit of ENZ plus ADT with ADT alone for men with mCSPC. ${ }^{9}$ Although the data for OS are immature and inadequate, radiographic progression-free survival (rPFS), the primary endpoint, was significantly better in the ENZ plus ADT group than in the ADT group (HR, 0.39; 95\% CI, 0.30-0.50; $\mathrm{p}<0.001$ ). This study recruited $\mathrm{mCSPC}$ patients irrespective of the tumor volume and previous chemotherapy with docetaxel; this is a unique point of this study. The oncologic benefit of upfront ENZ over ADT alone was consistent across all subgroups, including men with high-volume (HR, 0.43; 95\%
CI, 0.33-0.57) and low-volume (HR, 0.25; 95\% CI, 0.14-0.46) and those with (HR, 0.52; 95\% CI 0.30-0.89) or without prior docetaxel (HR, 0.37, 95\% CI, 0.28-0.49). Another trial, the Enzalutamide in First-Line Androgen-Deprivation Therapy for Metastatic Prostate Cancer (ENZAMET), compared the efficacy of upfront ENZ between patients receiving ENZ plus ADT and those receiving first-generation nonsteroidal antiandrogens (bicalutamide, flutamide, or nilutamide) plus ADT. ${ }^{10}$ Upfront ENZ was associated with significantly longer OS (HR, $0.67 ; 95 \%$ CI, 0.52-0.86; $\mathrm{p}=0.002$ ) and FFS (HR, 0.39; 95\% CI, 0.33-0.47; p < 0.001) than ADT plus first-generation nonsteroidal antiandrogen. In the subgroup analyses, the ENZ arm achieved longer progression-free survival (PFS) than the first-generation antiandrogen arm across all subgroups. ENZ was associated with better OS in patients with low-volume tumor burden and those without previous docetaxel use. Differences in the OS between the treatment groups did not reach statistical significance in patients with highvolume disease $(\mathrm{HR}=0.80 ; 95 \% \mathrm{CI}=0.5-1.07)$ and those with previous docetaxel (HR, 0.90; 95\% CI, 0.62-1.31), probably owing to the short follow-up period (median 34 mon); a longer follow-up is necessary for determining the effect of ENZ on OS in this trial.

Apalutamide (APA), another second-generation nonsteroidal antiandrogen, was assessed the efficacy for mCSPC in the Apalutamide Plus ADT Versus ADT in Patients With Metastatic Hormone-Sensitive Prostate Cancer (TITAN) study. ${ }^{11}$ This study included patients with low-volume and high-volume disease and those with previous docetaxel chemotherapy. Patients were randomized to treatment with APA plus ADT or placebo plus ADT and the primary end points were OS and rPFS. rPFS was significantly longer with the APA group than in the placebo group $(\mathrm{HR}=0.48 ; 95 \%$ $\mathrm{CI}=0.39-0.60 ; \mathrm{P}<0.001)$ and the effect of APA was consistently favorable across the subgroups, including tumor burden and previous docetaxel use. OS was also significantly longer in the APA group than in the placebo group (HR, 0.67; 95\% CI, 0.51-0.89; $\mathrm{p}=0.005$ ). Similar favorable tendency of OS was observed in the subgroup analyses, including tumor volume and previous docetaxel at an interim analysis performed at 24 mon.

These recent clinical trials suggest that early combination therapies are consistently associated with better outcomes than ADT alone, irrespective of the tumor burden and risk category. Thus, recent clinical guidelines recommend a combination of docetaxel or ARPIs with ADT as first-line therapy for all patients with mCSPC. ${ }^{13,14}$ The era of ADT alone may end and upfront combination therapies 
are becoming a standard of care as the initial treatment for "all-comers" with mCSPC.

\section{The Rationale for a Paradigm Shift from the "All-Comers" Toward the "Personalized" Approach Insufficient Evidence of Upfront Combination Therapies as Standard of Care for All Patients}

Several high-quality clinical trials have concluded that early combination therapies provided survival benefit for all men with mCSPC, as mentioned above. The clinical trials, however, excluded elderly subjects or patients with poor performance status (Table 1). The survival benefit and tolerability of upfront combination therapies are unknown in elderly and/or frail patients, and evidence regarding clinical benefit in these patient groups is lacking. The exploratory subgroup analyses failed to show any significant benefit of combination therapies over ADT alone in some specific patient groups, although favorable consistency was observed. The benefit was statistically unproven in several subgroups in terms of the Gleason score $(<8),{ }^{10,26}$ some regions, ${ }^{10,23}$ younger age $(<70 \mathrm{y}),{ }^{10}$ and older age $(>75 \mathrm{y}),{ }^{23}$ although low statistical power with small number of patients in each subgroup may have caused these non-significant results. Recent meta-analyses, including largescale clinical trials, have performed subgroup analysis. The meta-analysis by Sathianathen et al included six RCTs (ENZAMET, TITAN, GETUG-AFU-15, CHAARTED, STAMPEDE, and LATITUDE) with 8388 patients. $^{27}$ Addition of ENZ, APA, docetaxel, or AAP to ADT achieved better OS than ADT alone in patients with high-volume disease. In patients with low-volume disease, all the combination treatments showed tendency of favorable survival over ADT alone; however, only ENZ demonstrated significant OS benefit than ADT alone (HR, 0.38, 95\% CI, 0.20-0.68). The same results were also obtained in another meta-analysis. ${ }^{28}$ This meta-analysis included seven RCTs (ENZAMET, TITAN, GETUG-AFU-15, CHAARTED, STAMPEDE, LATITUDE, HORRAD) with 8820 patients. In the low-volume subgroup, ENZ, APA, docetaxel, AAP, and external beam radiation therapy (EBRT) to the prostate had superior OS than ADT alone; however, statistical significance was only observed for ENZ (HR, 0.38; 95\% CI, 0.21-0.69) and EBRT (HR, 0.68; $95 \%$ CI, 0.54-0.87). In patients with Gleason score $<8$, APA (HR, 0.56, 95\% CI, 0.33-0.95) and docetaxel (HR, 0.71; 95\% CI, 0.54-0.92) had significant OS benefit over ADT alone; however, others did not show this result. Therefore, upfront combination therapies may not be the standard of care for allcomers.

Table I Published Clinical Trials of Systemic Therapy for Metastatic Castration-Sensitive Prostate Cancer

\begin{tabular}{|c|c|c|c|c|c|c|c|}
\hline $\begin{array}{l}\text { Trial } \\
\text { Name }\end{array}$ & $\begin{array}{l}\text { Experimental } \\
\text { Arm }\end{array}$ & Control Arm & Risk & $\begin{array}{l}\text { Age: Median } \\
\text { (Yr, Range) }\end{array}$ & $\begin{array}{l}\text { Performance } \\
\text { Status }\end{array}$ & Results on Primary Endpoint(s) & Ref \\
\hline & & & & Experimental/Control Arm & & & \\
\hline CHAATED & DTX+ADT & ADT & All & $64(38-88) / 63(39-91)$ & $\mathrm{ECOG} \leq 2$ & $\begin{array}{l}\text { OS benefit for high-vol cancer No OS } \\
\text { benefit for low-vol cancer }\end{array}$ & {$[21]$} \\
\hline $\begin{array}{l}\text { GETUG- } \\
\text { AFU } 15\end{array}$ & $\mathrm{DTX}+\mathrm{ADT}$ & ADT & All & 63 (IQR 58-70)/64 (IQR 57-68) & $\begin{array}{l}\text { Karnofsky } \geq \\
70 \%\end{array}$ & No OS benefit & [22] \\
\hline $\begin{array}{l}\text { STAMPEDE } \\
\operatorname{arm} \mathrm{A} / \mathrm{C}\end{array}$ & $D T X+A D T$ & ADT & All & 65 (IQR 60-7I)/65 (IQR 60-70) & $\mathrm{WHO} \leq 2$ & OS benefit & [6] \\
\hline LATITUDE & $\mathrm{AAP}+\mathrm{ADT}$ & ADT+ placebo & High risk & $68(38-89) / 67(33-92)$ & $\mathrm{ECOG} \leq 2$ & rPFS and OS benefit & [23] \\
\hline $\begin{array}{l}\text { STAMPEDE } \\
\text { arms A/G }\end{array}$ & $A A P+A D T$ & ADT & All & 67(IQR 62-72)/67(IQR 62-72) & $\mathrm{WHO} \leq 2$ & OS benefit & [8] \\
\hline ENZAMET & ENZ+ADT & $\begin{array}{l}\mathrm{ADT}+\text { first-generation } \\
\text { antiandrogen }\end{array}$ & All & $69(63-75) / 69(63-75)$ & $E C O G \leq 2$ & OS benefit & {$[10]$} \\
\hline ARCHES & ENZ+ADT & ADT+ placebo & All & $70(46-92) / 70(42-92)$ & $E C O G \leq 1$ & rPFS benefit & [9] \\
\hline TITAN & $A P A+A D T$ & ADT+ placebo & All & $68(43-91) / 68(43-90)$ & $E C O G \leq 1$ & rPFS and $O S$ benefit & [II] \\
\hline
\end{tabular}

Abbreviations: AAP, abiraterone acetate and prednisone; ADT, androgen deprivation therapy; APA, apalutamide; DTX, docetaxel; ECOG, Eastern Cooperative Oncology Group; ENZ, enzalutamide; high-vol, high-volume; IQR, interquartile; low-vol, low-volume; OS, overall survival; rPFS, radiographic progression-free survival; WHO, World Health Organization. 


\section{Definitive Treatment is Beneficial Only in Patients with Low-Volume Disease}

Not only systemic pharmacotherapy, but radiation to the prostate also improves OS in low-volume disease. The recent high-quality RCTs, STAMPEDE ${ }^{29}$ and Hormonal Therapy Versus Hormonal Therapy Plus Local External Radiation Therapy in Patients With Primary Diagnosed Metastasized Prostate Cancer (HORRAD) ${ }^{30}$ concluded that adding radiation therapy to the prostate in $\mathrm{mCSPC}$ patients receiving ADT did not further improve their OS, the primary endpoint. In contrast, subgroup analyses by metastatic burden in the STAMPEDE trial showed OS benefit for patients with low-volume disease (HR, 0.68; 95\% CI, 0.52-0.90). The HORRAD trial also showed a similar trend without statistical significance in patients with $<5$ metastatic lesions. Meta-analysis of 2 RCTs that involved 2493 patients suggested that ADT plus EBRT to the prostate was associated with improved OS as compared to ADT alone in men with low-volume metastatic burden (HR, 0.68, 95\% CI, 0.54-0.87); however, this result was not observed in those with high-volume disease (HR, 1.1, 95\% CI, 0.92-1.2). ${ }^{28}$ Prostatectomy may also improve the oncologic outcomes in patients with oligometastatic prostate cancer. ${ }^{31}$ The definitive treatments, either radiation or prostatectomy, may be associated with survival benefit in patients with low metastatic burden. The results of several ongoing clinical trials on the benefit of prostatectomy and radiation to the prostate are expected to provide more information on this subject. ${ }^{32}$

\section{Balance of Efficacy and Toxicity}

Serious adverse events $(\geq$ grade 3 ) were observed in $30 \%$ $-60 \%$ of patients receiving combination therapies. ${ }^{9-11,23,25}$ The toxicity may exceed the benefits in some patients. Increased medical expense with combination therapies is also an issue.

Some metastatic patients are also shown to achieve longterm survival with ADT alone. For example, patients with lowvolume and low-risk mCSPC who achieved PSA $\leq 2 \mathrm{ng} / \mathrm{mL}$ at 3 mon after ADT commencement had a considerably long OS of 112 mon with ADT with or without first-generation antiandrogens. ${ }^{33}$ It is very likely that upfront combination therapy using docetaxel and ARPIs would be excessive for these patients. The profile of adverse events and medical cost are different among agents. For example, docetaxel is cheaper than ARPIs, but it is associated with neutropenia and peripheral neuropathy. ${ }^{5}$ ENZ increases the risk of fatigue, hypertension, cardiovascular event, and seizure. ${ }^{9,10,34} \mathrm{Abi}$ is associated with hypertension, cardiovascular events, and hypokalemia. ${ }^{23,34}$ Rash is relatively common adverse event of APA. ${ }^{11}$ Therefore, treatments must be tailored according to the individual patient's profile, comorbidities and preferences, and it is crucial to choose the appropriate treatments for each patient while avoiding unnecessary overtreatment. All-comers therapeutic approaches may not be used anymore in clinical practice.

\section{Future Perspective}

With advances in imaging modalities, treatment strategies are expected to evolve. Positron emission tomography (PET) of ${ }^{68}$ Ga-labelled prostate-specific membrane (PSMA-PET) has a very high detection rate for prostate cancer lesions. The sensitivity and specificity of PSMA-PET are $80 \%$ and $97 \%$, respectively, on per lesion analysis, and this tool is able to detect cancer lesions at very low levels of PSA. ${ }^{35}$ Highly sensitive imaging modalities are able to detect very tiny lesions that are not detected with conventional $\mathrm{CT}$ and bone scan and will induce stage migration. Therefore, definitions of cancer volume and risk category may change, and treatment strategies would be determined accordingly.

Prostate cancer has marked diversity in its biological and clinical features, probably determined by germline and somatic gene alteration as well as gene polymorphisms of the patients. Substantial number of single nucleotide polymorphisms are associated with OS and PFS in patients who receive ADT. ${ }^{36}$ These genetic diversities influence the treatment response and toxicity; moreover, the response to treatment would be different among patients even if they have similar cancer characteristics.

Investigation of the gene expression in the prostate cancer tissue can help identify promising predictive biomarkers. ${ }^{37,38}$ For example, the addition of docetaxel to ADT was beneficial for those with luminal B subtype of $\mathrm{mCSPC}$, determined by the expression profile of the 50 genes using PAM50; however, this was not beneficial for those with the basal subtype. ${ }^{38}$

Feng et $\mathrm{al}^{39}$ investigated the gene expression in primary prostate cancer patients enrolled in the TITAN study. The gene expression subtype was classified into DECIPHER genomic classifier $(\mathrm{GC})$ as high versus average-to-low risk of metastases, basal and luminal $\mathrm{A} / \mathrm{B}$ determined with PAM50, and androgen receptor activity (AR-A) signature high and low. The addition of APA to ADT improved the PFS in GC high-risk subtype (median PFS: not reached vs 18.2 mon; HR, 0.40; 95\% CI, 0.24-0.67; $p=0.0004)$, but not 
in GC average-to-low subtypes. APA also showed benefit in terms of PFS in patients with basal subtype (APA vs placebo; median PFS: not reached vs 18.4 mon; HR, 0.31; 95\% CI, $0.18-0.62 ; \mathrm{p}=0.0008$ ) and AR-A low subtype (APA vs placebo; median PFS: not reached vs 15.0 months; HR, $0.25 ; 95 \% \mathrm{CI}, 0.12-0.52 ; \mathrm{p}=0.0002$ ) disease. These results suggest that APA is beneficial in patients with specific molecular subtypes, but not in others. Alterations in germline DNA damage repair (DDR) genes, such as BRCA2 and $A T M$, predicted the response to ADT. Men with germline DDR gene mutations had significantly shorter time to progression to CRPC than those with wild type DDR genes (median time to CRPC; 8.3 vs 13.2 months; HR, 2.37; 95\% CI, 1.48-3.80; $\mathrm{p}<0.001) .{ }^{40}$ DDR gene status in circulating tumor DNA was also associated with progression to CRPC. Men harboring DDR gene mutations had shorter time to CRPC than those with wild type DDR genes (7.3 months vs not reached; $p=0.01) .{ }^{41}$ Patients with DDR gene mutations have poor response to $\mathrm{ADT}$ alone and combination of ADT with platinum-based chemotherapy ${ }^{42}$ or PARP inhibitors ${ }^{43}$ would be beneficial for patients with DDR gene mutations.

Androgen-androgen receptor signaling and other molecular pathways such as $\mathrm{Wnt} / \beta$-Catenin signaling and PTEN/PI3K/AKT/mTOR pathway ${ }^{44}$ are involved in hormone- and docetaxel-resistance. For example, AR splice variant, AR-V7, expression was associated with primary resistance to $\mathrm{Abi}$ or $\mathrm{ENZ}^{45,46}$ and $\mathrm{AR}-\mathrm{V} 7$ expression in CSPC was correlated with poor response to ADT. ${ }^{47}$ The SPOP gene was frequently mutated in primary prostate cancer and its mutations induced resistance to docetaxel. ${ }^{48}$

The molecular heterogeneity is associated with different clinical outcomes among patients with similar cancer characteristics. The genomic-driven approach will provide the precise predictive biomarkers that identify the subset of patients who would benefit most from the treatment. We will be able to tailor the optimal treatment for each patient, and treatments will shift from the "all-comers" to the "individualized" approach.

\section{Possible Future Treatment Strategy as per the Clinical and Biological Characteristics}

The treatment strategies should be determined as per cancer and patient characteristics as well as patient preference. We showed the possible treatment strategy for patients with mCSPC as per patient subgroups (Figure 1). Group 1: elderly and/or fragile patients with

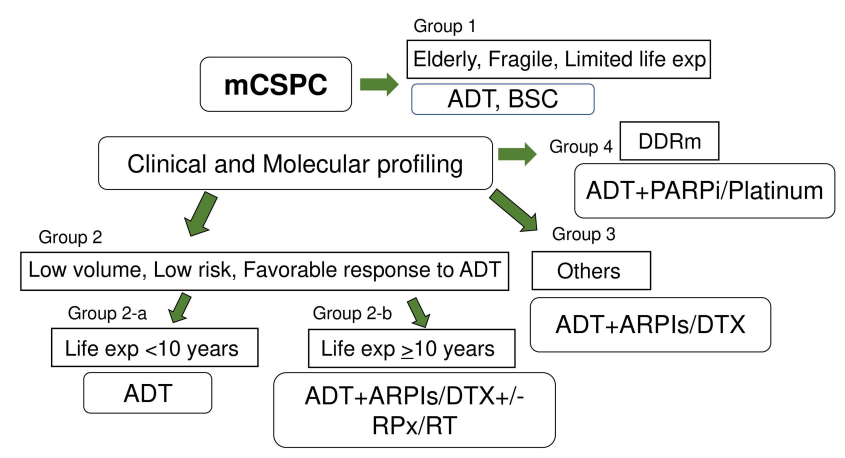

Figure I Perspective of treatment strategy for metastatic castration-sensitive prostate cancer as per the clinical and biological characteristics.

Abbreviations: ADT, androgen-deprivation therapy; ARPIs, androgen receptor pathway inhibitors; BSC, best supportive care; DDRm, DNA damage repair gene mutation; DTX, docetaxel; life exp, life expectancy; mCSPC, metastatic castrationsensitive prostate cancer; PARPi, poly (ADP-ribose) polymerase inhibitor; Platinum, platinum-based chemotherapy; RPx, radical prostatectomy; RT, radiation therapy.

very limited life expectancy; ADT alone or best supportive care. Group 2-a: patients with low-volume, low-risk disease, favorable response to ADT, and life expectancy $<10$ $\mathrm{y}$; ADT alone, because long-term OS, about 10 years, is expected with ADT alone in this patient group. ${ }^{33}$ Adverse events caused by upfront combination therapies may exceed their efficacy in those patients. Group 2-b: patients with the same cancer characteristics as group 2-a patients and having longer life expectancy ( $>10 \mathrm{y}$ ); upfront combination therapies using docetaxel or ARPIs, with or without prostatectomy or EBRT to the prostate together with ADT. Metastases-directed radiotherapy may be also indicated. Complete eradication of cancer and cure may be anticipated with these aggressive combination treatments in these patients. Group 3: patients with high-volume, highrisk, or unfavorable response to ADT; upfront combination therapies using docetaxel or ARPIs. Group 4: patients carrying DDR gene mutations; poly (ADP-ribose) polymerase (PARP) inhibitors or platinum-based chemotherapy. The prognostic and predictive biomarker-based decision will enable optimal personalized treatment.

\section{Conclusion}

There has been remarkable evolution in MCSPC treatment in the previous several years. Recent upfront combination treatments with ADT have improved the oncologic outcomes and are becoming a standard of care for mCSPC. Treatments should be tailored to each patient because $\mathrm{mCSPC}$ has marked diversity in its biological and clinical features. Recent advance in the field of imaging and molecular technology will allow more effective individualized treatment, improving the changes of favorable clinical outcomes. 


\section{Acknowledgments}

The authors would like to thank Enasgo for the English language review.

\section{Disclosure}

The authors report no conflicts of interest in this work.

\section{References}

1. Siegel RL, Miller KD, Jemal A. Cancer statistics, 2020. CA Cancer J Clin. 2020;70(1):7-30. doi:10.3322/caac.21590

2. Teoh JYC, Hirai HY, Ho JM, et al. Global incidence of prostate cancer in developing and developed countries with changing age structures. PLoS One. 2019;14(10):e0221775. doi:10.1371/journal.pone.0221775

3. Sharifi NJ, Gulley JL, Dahut WL. An update on androgen deprivation therapy for prostate cancer. Endocr Relat Cancer. 2010;17(4):R30515. doi:10.1677/ERC-10-0187

4. James ND, Spears MR, Clarke NW, et al. Survival with newly diagnosed metastatic prostate cancer in the "docetaxel era": data from 917 patients in the control arm of the STAMPEDE trial (MRC PR08, CRUK/06/019). Eur Urol. 2015;67(6):1028-1038. doi:10.1016/j.eururo.2014.09.032

5. Sweeney CJ, Chen YH, Carducci M, et al. Chemohormonal therapy in metastatic hormone-sensitive prostate cancer. $N$ Engl $\mathrm{J}$ Med. 2015;373(8):737-746. doi:10.1056/NEJMoa1503747

6. James ND, Sydes MR, Clarke NW, et al. Addition of docetaxel, zoledronic acid, or both to first-line long-term hormone therapy in prostate cancer (STAMPEDE): survival results from an adaptive, multiarm, multistage, platform randomised controlled trial. Lancet. 2016;387(10024):1163-1177. doi:10.1016/S0140-6736(15)01037-5

7. Fizazi K, Tran N, Fein L, et al. Abiraterone plus prednisone in metastatic, castration-sensitive prostate cancer. $N$ Engl $J$ Med. 2017;377(4):352-360. doi:10.1056/NEJMoa1704174

8. James ND, de Bono JS, Spears MR, et al. Abiraterone for prostate cancer not previously treated with hormone therapy. $N$ Engl $J$ Med. 2017;377(4):338-351. doi:10.1056/NEJMoa1702900

9. Armstrong AJ, Szmulewitz RZ, Petrylak DP, et al. ARCHES: a randomized, phase iii study of androgen deprivation therapy with enzalutamide or placebo in men with metastatic hormone-sensitive prostate cancer. $J$ Clin Oncol. 2019;37(32):2974-2986. doi:10.1200/JCO.19.00799

10. Davis ID, Martin AJ, Martin SR, et al. Enzalutamide with standard first-line therapy in metastatic prostate cancer. $N$ Engl $J$ Med. 2019;381(2):121-131. doi:10.1056/NEJMoa1903835

11. Chi KN, Agarwal N, Bjartell A, et al. Apalutamide for metastatic castration-sensitive prostate cancer. $N$ Engl J Med. 2019;381(1):1324. doi:10.1056/NEJMoa1903307

12. Hoyle AP, Ali A, James ND, et al. Abiraterone in "high-" and "lowrisk" metastatic hormone-sensitive prostate cancer. Eur Urol. 2019;76 (6):719-728. doi:10.1016/j.eururo.2019.08.006

13. Cornford P, van den Bergh RCN, Briers E, et al. EAU-EANMESTRO-ESUR-SIOG guidelines on prostate cancer. Part II-2020 update: treatment of relapsing and metastatic prostate cancer. Eur Urol. 2021;79(2):263-282. doi:10.1016/j.eururo.2020.09.046

14. Lowrance WT, Breau RH, Chou R, et al. Advanced prostate cancer: AUA/ASTRO/SUO guideline PART I. J Urol. 2020;205(1-8).

15. Damodaran S, Lang JM, Jarrard DF, et al. Targeting metastatic hormone sensitive prostate cancer: chemohormonal therapy and new combinatorial approaches. $J$ Urol. 2019;201(5):876-885. doi:10.1097/JU.0000000000000117

16. VanderWeele DJ, Antonarakis ES, Carducci MA, et al. Metastatic hormone-sensitive prostate cancer: clinical decision making in a rapidly evolving landscape of life-prolonging therapy. J Clin Oncol. 2019;37(32):2961-2967. doi:10.1200/JCO.19.01595
17. Barata PC, Sartor AO. Metastatic castration-sensitive prostate cancer: abiraterone, docetaxel, or. Cancer. 2019;125(11):1777-1788. doi:10.1002/cncr.32039

18. Parker DC, Cookson MS. The changing landscape in the management of newly diagnosed castration sensitive metastatic prostate cancer. Investig Clin Urol. 2020;61(Suppl 1):S3-S7. doi:10.4111/ icu.2020.61.S1.S3

19. Hall ME, Huelster HL, Luckenbaugh AN, et al. Metastatic hormonesensitive prostate cancer: current perspective on the evolving therapeutic landscape. Onco Targets Ther. 2020;13:3571-3581. doi:10.2147/OTT.S228355

20. Laccetti AL, Morris MJ, Kantoff PW. A clinical evaluation of enzalutamide in metastatic castration-sensitive prostate cancer: guiding principles for treatment selection and perspectives on research. Onco Targets Ther. 2020;13:13247-13263. doi:10.2147/OTT.S242921

21. Kyriakopoulos CE, Chen YH, Carducci MA, et al. Chemohormonal therapy in metastatic hormone-sensitive prostate cancer: long-term survival analysis of the randomized phase III E3805 CHAARTED trial. J Clin Oncol. 2018;36(11):1080-1087. doi:10.1200/JCO.2017.75.3657

22. Gravis G, Boher JM, Chen YH, et al. Burden of metastatic castrate naive prostate cancer patients, to identify men more likely to benefit from early docetaxel: further analyses of CHAARTED and GETUGAFU15 studies. Eur Urol. 2018;73(6):847-855. doi:10.1016/j. eururo.2018.02.001

23. Fizazi K, Tran N, Fein L, et al. Abiraterone acetate plus prednisone in patients with newly diagnosed high-risk metastatic castration-sensitive prostate cancer (LATITUDE): final overall survival analysis of a randomised, double-blind, phase 3 trial. Lancet Oncol. 2019;20 (5):686-700. doi:10.1016/S1470-2045(19)30082-8

24. Vale CL, Burdett S, Rydzewska LHM, et al. Addition of docetaxel or bisphosphonates to standard of care in men with localised or metastatic, hormone-sensitive prostate cancer: a systematic review and meta-analyses of aggregate data. Lancet Oncol. 2016;17(2):243256. doi:10.1016/S1470-2045(15)00489-1

25. Clarke NW, Ali A, Ingleby FC, et al. Addition of docetaxel to hormonal therapy in low- and high-burden metastatic hormone-sensitive prostate cancer: long-term survival results from the STAMPEDE trial. Ann Oncol. 2019;30(12):1992-2003. doi:10.1 093/annonc/mdz396

26. Rydzewska LHM, Burdett S, Vale CL, et al. Adding abiraterone to androgen deprivation therapy in men with metastatic hormone-sensitive prostate cancer: a systematic review and meta-analysis. Eur $J$ Cancer. 2017;84:88-101. doi:10.1016/j.ejca.2017.07.003

27. Sathianathen NJ, Koschel S, Thangasamy IA, et al. Indirect comparisons of efficacy between combination approaches in metastatic hormonesensitive prostate cancer: a systematic review and network meta-analysis. Eur Urol. 2020;77(3):365-372. doi:10.1016/j.eururo.2019.09.004

28. Wang Y, Gui H, Wang J, et al. Comparative efficacy of combined radiotherapy, systemic therapy, and androgen deprivation therapy for metastatic hormone-sensitive prostate cancer: a network meta-analysis and systematic review. Front Oncol. 2020;10:567616. doi: $10.3389 /$ fonc. 2020.567616

29. Parker CC, James ND, Brawley CD, et al. Radiotherapy to the primary tumour for newly diagnosed, metastatic prostate cancer (STAMPEDE): a randomised controlled phase 3 trial. Lancet. 2018;392(10162):2353-2366. doi:10.1016/S0140-6736(18)32486-3

30. Boevé LMS, Hulshof MCCM, Vis AN, et al. Effect on survival of androgen deprivation therapy alone compared to androgen deprivation therapy combined with concurrent radiation therapy to the prostate in patients with primary bone metastatic prostate cancer in a prospective randomised clinical trial: data from the HORRAD trial. Eur Urol. 2019;75(3):410-418. doi:10.1016/j.eururo.2018.09.008

31. Gandaglia G, Fossati N, Stabile A, et al. Radical prostatectomy in men with oligometastatic prostate cancer: results of a single-institution series with long-term follow-up. Eur Urol. 2017;72(2):289-292. doi:10.1016/j.eururo.2016.08.040 
32. Ranasinghe W, Chapin BF, Kim IY, Sooriakumaran P, Lawrentschuk N. The cytoreductive prostatectomy in metastatic prostate cancer: what the individual trials are hoping to answer. BJU Int. 2020;125 (6):792-800. doi:10.1111/bju.15055

33. Fujimoto N, Shiota M, Matsukawa T, et al. Three-month prostatespecific antigen level after androgen deprivation therapy predicts survival in patients with metastatic castration-sensitive prostate cancer. In vivo. 2021;35(2):1101-8.

34. Iacovelli R, Ciccarese C, Bria E, et al. The cardiovascular toxicity of abiraterone and enzalutamide in prostate cancer. Clin Genitourin Cancer. 2018;16(3):e645-e653. doi:10.1016/j.clgc.2017.12.007

35. Perera M, Papa N, Christidis D, et al. Sensitivity, specificity, and predictors of positive $68 \mathrm{Ga}$-prostate-specific membrane antigen positron emission tomography in advanced prostate cancer: a systematic review and meta-analysis. Eur Urol. 2016;70(6):926-937. doi:10.1016/j.eururo.2016.06.021

36. Fujimoto N, Shiota M, Tomisaki I, Minato A. Gene polymorphismrelated individual and interracial differences in the outcomes of androgen deprivation therapy for prostate cancer. Clin Genitourin Cancer. 2017;15(3):337-342. doi:10.1016/j.clgc.2017.01.006

37. Zhao SG, Chang SL, Erho N, et al. Associations of luminal and basal subtyping of prostate cancer with prognosis and response to androgen deprivation therapy. JAMA Oncol. 2017;3(12):1663-1672. doi:10.1001/jamaoncol.2017.0751

38. Hamid A, Wang XV, Chen YH, et al. Outcomes, responses to therapy vary based on basal-luminal subtype in metastatic prostate cancer. Genitourinary cancers symposium. Abstract \#162. Available from: https://meetinglibrary.asco.org/record/184248/abstract. Accessed March 25, 2021.

39. Feng FY, Thomas S, Aguilar-Bonavides C, Gormley M, Agarwal N, Attard G. Molecular determinants of outcome for metastatic castration-sensitive prostate cancer ( $\mathrm{mCSPC}$ ) with addition of apalutamide (APA) or placebo (PBO) to androgen deprivation therapy (ADT) in TITAN. $J$ Clin Oncol. 2020;38(15_suppl):Abstract \#5535. doi:10.1200/JCO.2020.38.15_suppl.5535
40. Wei Y, Wu J, Gu W, et al. Prognostic value of germline DNA repair gene mutations in de novo metastatic and castration-sensitive prostate cancer. Oncologist. 2020;25(7):e1042-e1050. doi:10.1634/theoncologist.2019-0495

41. Vandekerkhove G, Struss WJ, Annala M, et al. Circulating tumor DNA abundance and potential utility in de novo metastatic prostate cancer. Eur Urol. 2019;75(4):667-675. doi:10.1016/j.eururo.201 8.12 .042

42. Slootbeek PHJ, Duizer ML, van der Doelen MJ, et al. Impact of DNA damage repair defects and aggressive variant features on response to carboplatin-based chemotherapy in metastatic castration-resistant prostate cancer. Int J Cancer. 2021;148(2):385-395. doi:10.1002/ ijc. 33306

43. de Bono J, Mateo J, Fizazi K, et al. Olaparib for metastatic castrationresistant prostate cancer. $N$ Engl J Med. 2020;382(22):2091-2102. doi:10.1056/NEJMoa1911440

44. Pisano C, Tucci M, Di Stefano RF, et al. Interactions between androgen receptor signaling and other molecular pathways in prostate cancer progression: current and future clinical implications. Crit Rev Oncol Hematol. 2021;157:103185. doi:10.1016/j.critrevonc.20 20.103185

45. Efstathiou E, Titus M, Wen S, et al. Molecular characterization of enzalutamide-treated bone metastatic castration-resistant prostate cancer. Eur Urol. 2015;67(1):53-60. doi:10.1016/j.eururo.20 14.05.005

46. Antonarakis ES, Lu C, Wang $\mathrm{H}$, et al. AR-V7 and resistance to enzalutamide and abiraterone in prostate cancer. $N$ Engl $\mathrm{J} \mathrm{Med}$. 2014;371(11):1028-1038. doi:10.1056/NEJMoa1315815

47. Li H, Zhang Y, Li D, et al. Androgen receptor splice variant 7 predicts shorter response in patients with metastatic hormone-sensitive prostate cancer receiving androgen deprivation therapy. Eur Urol. 2021;S0302-2838(21)00078-6.

48. Shi Q, Zhu Y, Ma J, et al. Prostate cancer-associated SPOP mutations enhance cancer cell survival and docetaxel resistance by upregulating caprin1-dependent stress granule assembly. Mol Cancer. 2019;18 (1):170. doi:10.1186/s12943-019-1096-x

\section{Publish your work in this journal}

OncoTargets and Therapy is an international, peer-reviewed, open access journal focusing on the pathological basis of all cancers, potential targets for therapy and treatment protocols employed to improve the management of cancer patients. The journal also focuses on the impact of management programs and new therapeutic agents and protocols on patient perspectives such as quality of life, adherence and satisfaction. The manuscript management system is completely online and includes a very quick and fair peer-review system, which is all easy to use. Visit http://www.dovepress.com/ testimonials.php to read real quotes from published authors. 